\title{
A micromechanical model of influence of particle fracture and particle cluster on mechanical properties of metal matrix composites
}

\author{
G.K. Hu ${ }^{\text {a,b. * }}$, G. Guo ${ }^{\circ}$, D. Baptiste ${ }^{c}$ \\ a Department of Applied Mechanics, Beijing Institute of Technology, 10008I Beijing, PR China \\ ${ }^{\mathrm{h}}$ LNM Institute of Mechanics Academia Sinica. 100080 Beijing, PR China \\ ' LM3 ENSAM, 151. Boulevard de L'Hopital, 75013 Paris, France
}

Received 18 July 1997; accepted 19 August 1997

\begin{abstract}
A general analytical model for a composite with an isotropic matrix and two populations of spherical inclusions is proposed. The method is based on the second order moment of stress for evaluating the homogenised effective stress in the matrix and on the secant moduli concept for the plastic deformation. With Webull's statistical law for the strength of $\mathrm{SiCp}$ particles, the model can quantitatively predict the influence of particle fracture on the mechanical properties of PMMCs. Application of the proposed model to the particle cluster shows that the particle cluster has neglected influence on the strain and stress curves of the composite. (C) 1998 Elsevier Science B.V.
\end{abstract}

\section{Introduction}

Particle reinforced light metals, with their potential as low cost, high modulus and strength and easily fabricated materials, have received particular attention in the past decade [1]. Much efforts have been devoted to the understanding of the strengthening mechanisms for such materials [2-4]. It is now known that the overall elastoplastic behaviour of particle reinforced metal matrix composites (PMMCs) can be modelled using a continuum approach, for example, the periodic cell model $[2,4]$ or the Eshelby equivalent inclusion method with secant moduli approach [5-8].

The major limitations in the mechanical properties of the PMMCs is the rather limited ductility and the fact that the composite failure strain decreases rapidly with the addition of the reinforcing particles. The final fracture of the composite is believed to be the consequence of the damage initiation, its accumulation and coalescence during loading. For $\mathrm{SiCp} / \mathrm{Al}$ composite systems, the main damage mechanism consists of the particle fracture $[1,4,7,10]$. The particles with high aspect ratio or volume are more easily fractured and these

\footnotetext{
' Corresponding author. Fax: + 86-10-68913205.
} 
effects can be explained by Weibull statistical distribution for the strength of $\mathrm{SiCp}$ and the load transferring mechanism between the particle and matrix $[1,4,7,8]$.

Due to the fabrication process, PMMCs often present non-uniform distribution of the reinforced particles, forming particle cluster zones. The formation of these particle cluster zones, may have an influence on the plastic behaviour, especially on damage initiation and development of the composites. In addition, the matrix in the cluster zones is subjected to a high triaxial stress state and this may promote void formation in these zones.

All these factors are important when the failure strain of the composites are addressed.

In this paper, we will present a simple analytical model to account for the effects mentioned above (particle fracture and particle cluster). The modelled composite is assumed to consist of two populations of spherical inclusions, for the purpose of modelling the effects of particle fracture and of particle cluster. An homogeniscd matrix effective stress, based on the distortion energy [11] or second order moment of stress [12,13] will be evaluated in an analytical form. The plastic deformation of the matrix will be taken into account by the secant moduli method [5,6]. The general formulation will then be applied to investigate the particle fracture by considering the broken particles as voids and to study the influence of particle cluster by taking it as a composite inclusion. Since the essential step for the secant moduli method consists of using a linear comparison composite, whose matrix moduli is set equal to the secant moduli of the studied composite, we will present first the results for an elastic composite.

\section{Theoretical consideration}

\subsection{Elastic moduli for a composite with two populations of inclusions}

The considered composite is assumed to consist of an isotropic continue matrix characterised by $\mu_{0}, k_{0}$ and $c_{0}$ (shear, bulk moduli and its volume fraction, respectively) and two populations of isotropic spherical inclusions (characterised by $\mu_{1}, k_{1}, c_{1}$ and $\mu_{2}, k_{2}, c_{2}$ ). The composite as a whole is isotropic. A representative volume element of the considered composite is now subjected to a macroscopic load $\Sigma_{i j}$, which is split into deviatoric and hydrostatic parts $\Sigma_{i j}=\Sigma_{i j}^{\prime}+\Sigma \delta_{i j}$. Following the Mori-Tanaka mean stress method [14] (see Appendix A), we can determine the average stress in the different phases and in turn obtain the shear and bulk moduli for the studied composite. The results for the shear modulus of the composite are

$$
\mu_{c}=\frac{a_{0}+a_{1} \mu_{1}+a_{2} \mu_{2}+a_{3} \mu_{1} \mu_{2}}{b_{0}+b_{1} \mu_{1}+b_{2} \mu_{2}+b_{3} \mu_{1} \mu_{2}} \mu_{0}
$$

where the constants are independent of the particles' properties, and

$$
\begin{aligned}
& a_{0}=81 c_{0} k_{0}^{2} \mu_{0}^{2}+144 c_{0} k_{0} \mu_{0}^{3}+64 c_{0} \mu_{0}^{4} \\
& a_{1}=\mu_{0}\left(9 k_{0}+8 \mu_{0}\right)\left[3\left(2 c_{0}+5 c_{1}\right) k_{0}+4\left(3 c_{0}+5 c_{1}\right) \mu_{0}\right] \\
& a_{2}=\mu_{0}\left(9 k_{0}+8 \mu_{0}\right)\left[3\left(2 c_{0}+5 c_{2}\right) k_{0}+4\left(3 c_{0}+5 c_{2}\right) \mu_{0}\right] \\
& a_{3}=6\left(k_{0}+2 \mu_{0}\right)\left[3\left(5-3 c_{0}\right) k_{0}+4\left(5-2 c_{0}\right) \mu_{0}\right]
\end{aligned}
$$

and

$$
\begin{aligned}
& b_{0}=27\left(5-2 c_{0}\right) k_{0}^{2} \mu_{0}^{2}+12\left(25-13 c_{0}\right) k_{0} \mu_{0}^{3}+32\left(5-3 c_{0}\right) \mu_{0}^{4} \\
& b_{1}=6 \mu_{0}\left(k_{0}+2 \mu_{0}\right)\left[3\left(3 c_{0}+5 c_{2}\right) k_{0}+4\left(2 c_{0}+5 c_{2}\right) \mu_{0}\right] \\
& b_{2}=6 \mu_{0}\left(k_{0}+2 \mu_{0}\right)\left[3\left(3 c_{0}+5 c_{1}\right) k_{0}+4\left(2 c_{0}+5 c_{1}\right) \mu_{0}\right] \\
& b_{3}=36 c_{0}\left(k_{0}+2 \mu_{0}\right)^{2}
\end{aligned}
$$


for the bulk modulus of the composite

$$
k_{c}=\frac{d_{0}+d_{1} k_{1}+d_{2} k_{2}+d_{3} k_{1} k_{2}}{e_{0}+e_{1} k_{1}+e_{2} k_{2}+e_{3} k_{1} k_{2}} k_{0}
$$

where $d_{0}=16 c_{0} \mu_{0}^{2}, \quad d_{1}=4 \mu_{0}\left[3 c_{0}+c_{1}\left(3+4 \mu_{0} / k_{0}\right)\right], \quad d_{2}=4 \mu_{0}\left[3 c_{0}+c_{2}\left(3+4 \mu_{0} / k_{0}\right)\right], \quad d_{3}=3[3+4(1-$ $\left.\left.\left.c_{0}\right) \mu_{0} / k_{0}\right)\right]$ and $e_{0}=12\left(1-c_{0}\right) \mu_{0} k_{0}+16 \mu_{0}^{2}, e_{1}=9 c_{2} k_{0}+12\left(1-c_{1}\right) \mu_{0}, e_{2}=9 c_{1} k_{0}+12\left(1-c_{2}\right) \mu_{0}$ and $e_{3}=9 c_{0}$.

\subsection{Homogenised matrix effective stress and stresses in the particles}

The homogeniscd matrix effective stress used in this paper is calculated directly from the average of its local value [11-13], this homogenised matrix effective stress is believed to account better for the local stress fluctuation in the matrix than that derived directly from the matrix average stress [11]. According to the results given by $\mathrm{Hu}$ [13], together with the expression of the composite shear and bulk moduli given by Eqs. (1) and (2), the homogenised matrix effective stress can be expressed as

$$
\sigma_{\text {eff }}^{2}=\frac{3}{2}\left\langle\sigma_{i j}^{\prime} \sigma_{i j}^{\prime}\right\rangle_{0}=\frac{1}{c_{0}}\left\{\left(\frac{\mu_{0}}{\mu_{c}}\right)^{2} \frac{\partial \mu_{c}}{\partial \mu_{0}} \Sigma_{i}^{2}+3\left(\frac{\mu_{0}}{k_{c}}\right)^{2} \frac{\partial k_{c}}{\partial \mu_{0}} \Sigma^{2}\right\}=\frac{\Sigma_{i}^{2}}{A^{2}}+\frac{\Sigma^{2}}{B^{2}}
$$

where $\Sigma_{i}^{2}=3 / 2 \Sigma_{i j}^{\prime} \Sigma_{i j}^{\prime}$ is the effective macroscopic stress. $\langle\cdot\rangle_{0}$ means the volume average of the said quantity over the matrix, $\sigma_{i j}^{\prime}$ is the stress deviator.

The constants $A$ and $B$ are

$$
\begin{aligned}
& \frac{1}{A^{2}}=\frac{h_{0}+h_{1} \mu_{1}+h_{2} \mu_{2}+h_{3} \mu_{1} \mu_{2}+h_{4} \mu_{1}^{2}+h_{5} \mu_{2}^{2}+h_{6} \mu_{2} \mu_{1}^{2}+h_{7} \mu_{1} \mu_{2}^{2}+h_{8} \mu_{1}^{2} \mu_{2}^{2}}{c_{0}\left(a_{0}+a_{1} \mu_{1}+a_{2} \mu_{2}+a_{3} \mu_{1} \mu_{2}\right)^{2}} \\
& \frac{1}{B^{2}}=\frac{3\left(g_{0}+g_{1} k_{1}+g_{2} k_{2}+g_{3} k_{1} k_{2}+g_{4} k_{1}^{2}+g_{5} k_{2}^{2}+g_{6} k_{1}^{2} k_{2}+g_{7} k_{1} k_{2}^{2}+g_{8} k_{1}^{2} k_{2}^{2}\right)}{c_{0}\left(d_{0}+d_{1} k_{1}+d_{2} k_{2}+d_{3} k_{1} k_{2}\right)^{2}}
\end{aligned}
$$

The constants in the above expressions are independent of the particle properties and are given in Appendix B.

By setting this homogenised matrix effective stress to the yield stress of the matrix, the yield surface of the analysed composite can be derived as

$$
\frac{\Sigma_{c}^{2}}{A^{2}}+\frac{\Sigma^{2}}{B^{2}}=\sigma_{y}^{2}
$$

where $\sigma_{y}$ is the yield stress for the matrix.

For the stresses in the inclusions, it was checked that the homogenised effective stress in the inclusions can be evaluated directly from the average stresses in the inclusions. So in the following only the relationships between the average stresses in the inclusion and the applied macroscopic stress are provided.

For the deviatoric stresses in the inclusions, they can be expressed as

$$
\begin{aligned}
& \sigma l_{i j}^{\prime}=\frac{5\left(3 k_{0}+4 \mu_{0}\right)\left[9 k_{0} \mu_{0}+8 \mu_{0}^{2}+\left(6 k_{0}+12 \mu_{0}\right) \mu_{2}\right]}{\left(a_{0}+a_{1} \mu_{1}+a_{2} \mu_{2}+a_{3} \mu_{1} \mu_{2}\right)} \mu_{1} \Sigma_{i j}^{\prime} \\
& \sigma 2_{i j}^{\prime}=\frac{5\left(3 k_{0}+4 \mu_{0}\right)\left[9 k_{0} \mu_{0}+8 \mu_{0}^{2}+\left(6 k_{0}+12 \mu_{0}\right) \mu_{1}\right]}{\left(a_{0}+a_{1} \mu_{1}+a_{2} \mu_{2}+a_{3} \mu_{1} \mu_{2}\right)} \mu_{2} \Sigma_{i j}^{\prime}
\end{aligned}
$$


for the hydrostatic stresses in the inclusions, they are

$$
\begin{aligned}
& \sigma 1=\frac{\left(3+4 \mu_{0} / k_{0}\right)\left(4 \mu_{0}+3 k_{2}\right)}{d_{0}+d_{1} k_{1}+d_{2} k_{2}+d_{3} k_{1} k_{2}} k_{1} \Sigma \\
& \sigma 2=\frac{\left(3+4 \mu_{0} / k_{0}\right)\left(4 \mu_{0}+3 k_{1}\right)}{d_{0}+d_{1} k_{1}+d_{2} k_{2}+d_{3} k_{1} k_{2}} k_{2} \Sigma
\end{aligned}
$$

Where $\sigma 1_{i j}=\sigma 1_{i j}^{\prime}+\sigma 1 \delta_{i j}, \sigma 2_{i j}=\sigma 2_{i j}^{\prime}+\sigma 2 \delta_{i j}$, are the stresses in the inclusions of the population one and of the population two.

The previous results are of a general nature, all expressions above are derived by the Mathematica program. The expressions are arranged in the form that the special composite systems can be easily analysed in a straightforward manner. For example the previous results are reduced to the case for one population of inclusion by setting the properties of the other population of the inclusions to zero. The rigid inclusion reinforced composite or porous materials can also be easily studied with above analytical expressions.

In the following, we will utilise the previous formulation to analyse the influence of particle fracture and the particle cluster on the overall behaviours for PMMCs.

\section{Application to PMMCs}

\subsection{Plastic deformation of the matrix}

Now the particles are assumed to be elastic and plastic deformation can occur in the matrix during a mechanical loading. The weakened constraint power by the plastic deformation of the matrix on the inclusion will be taken into account by the secant moduli concept [5,6]. For a matrix following a power type hardening law such as $\sigma_{\mathrm{y}}=h\left(\varepsilon_{0}+\varepsilon_{\mathrm{p}}\right)^{n}$, the secant shear and bulk moduli of the matrix can be written as

$$
\mu_{0}^{s}=1 /\left(1 / \mu_{0}+3 \varepsilon_{\mathrm{p}} /\left(h\left(\varepsilon_{0}+\varepsilon_{\mathrm{p}}\right)^{n}\right)\right), \quad k_{0}^{s}=k_{0}
$$

In Eq. (9) the plastic incompressibility of the matrix is assumed. Using the secant moduli concept, the previous elastic formulations derived in Section 2 can be generalised directly to the elasto-plastic cases. To this end the matrix shear and bulk moduli must be considered as the corresponding secant ones and so the effective moduli of the composite. A simple iteration is needed to determine for a given applied stress $\Sigma_{i j}$ the corresponding effective plastic strain $\varepsilon_{\mathrm{p}}$ in the matrix and this can be performed by equating the homogenised matrix effective stress (defined by Eq. (3)) to its current yield stress.

\subsection{Modelling of composite damage in form of particle fracture}

\subsubsection{Particle fracture}

Fracture of the SiCp during deformation of the $\mathrm{MMC}$ is usually observed for $\mathrm{SiCp} / \mathrm{Al}$ composite system $[1,4,7]$. The fractured inclusions will lose their load carrying capacity in the sense of tensile loading and this leads to an increase of the stress in the surrounding matrix. In order to include this effect in the model, we consider, as the first approximation, the fractured particles as voids of the same form as the broken particle. This method is schematically shown in Fig. 1 .

In this situation we take $\mu_{2}=0$ and $k_{2}=0$. The fracture criterion for the ceramic particles is assumed to be controlled by the maximum principle tensile stress in the inclusion, which is noted by $\sigma 1_{p l}$ and the strength of 


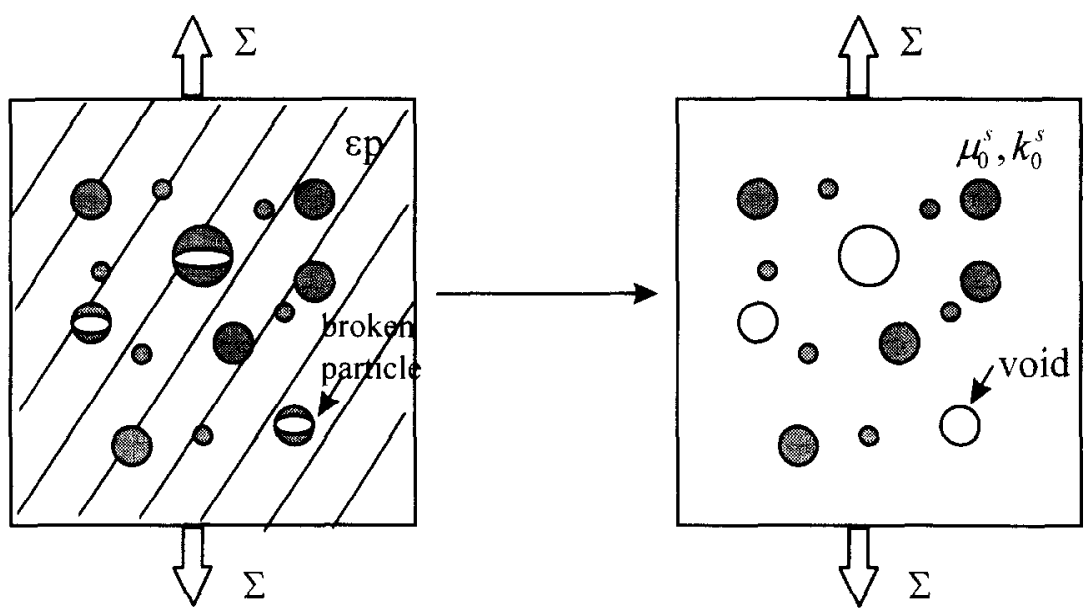

Fig. 1. Damage consideration for the particle fracture.

the ceramic particle is considered to obey the Weibull statistical law. For the analysed composite, the statistical distribution functions $p(V)$ for the SiCp reinforced phase are given by Llorca and Poza [9]

$$
p(V)=\frac{4 V}{\bar{V}^{2}} \exp \left(\frac{-2 V}{\bar{V}}\right)
$$

where $\bar{V}$ stands for the average reinforcement volume, $V$ is the volume of the particle. The volume fraction of broken inclusions reads [9]

$$
\frac{c_{2}}{f}=1-\frac{1}{\left[1+\frac{\bar{V}}{2 V_{0}}\left(\frac{\sigma 1_{p 1}-\sigma_{0}}{\sigma_{\mu}}\right)^{m}\right]^{2}}
$$

Where $f$ is the volume fraction of the initial particle and $c_{3}+c_{2}=f, \sigma_{0}$ is the threshold for the fracture of the particles, $V_{0}$ and $\sigma_{\mathrm{u}}$ are two constants introduced for dimensional purposes and $m$ is the Weibull modulus.

\subsubsection{Calculation results}

In the following calculation, only the uniaxial loading is considered. To perform the computation, we need to determine under a given load, $\Sigma_{11}$, the corresponding effective matrix plastic strain, $\varepsilon_{\mathrm{p}}$, and the volume fraction of the broken particles, $c_{2}$. This needs two iteration processes which can be achieved with the help of Eqs. (4) and (11). Once these relationships are determined, the elasto-plastic behaviour (including the damage) and the modulus variation of the composite can then be derived using the secant moduli method. This method is applied to a composite with two kinds of heat treatments called T4 and T621 (detailed conditions can be found in Ref. [4]). The material constants for the matrices and inclusions used in the computation are given in Table 1.

The volume fraction of the inclusion is 15\%. The results are shown in Figs. 2 and 3 for the overall elasto-plastic stress-strain relation and the modulus variation of the composite.

It can be seen that the predictions concerning the composite stress strain relation and modulus evolution are in quantitative agreement with the experiments. The prediction of the stress-strain curves for the composite materials is a little underestimated compared to the experimental results, this may come from the fact that in the experiment, the aspect ratio of $\mathrm{SiCp}$ is in fact a little larger than unity. 
Table 1

Material constants

\begin{tabular}{llllll}
\hline & $E_{\mathrm{m}}$ & $\nu_{\mathrm{m}}$ & $h$ & $\varepsilon_{0}$ & $n$ \\
\hline $\mathrm{T} 4$ & 70 & 0.33 & 0.628 & 0.00135 & 0.157 \\
$\mathrm{~T} 651$ & 70 & 0.33 & 0.535 & 0.035 & 0.07 \\
\hline $\mathrm{SiCp}$ & $E_{1}$ & $\nu_{1}$ & $\sigma_{0}$ & $\sigma_{\mathrm{u}}$ & $m$ \\
\hline
\end{tabular}

Unit for stress is $\mathrm{GPa}$.

\subsection{Modelling of the particle cluster in the composite}

Now we will consider the case in which the studied composite consists of the zones where the particle concentrations are different from that of rest media, this is schematically shown in Fig. 4. These zones, called the clusters of the particles, are assumed to be homogeneously distributed in the composite and to have a spherical form. A more general case will be considered by Guo et al. [15] in a general formulation.

In our model, local particle concentrations in the cluster zones are assumed to be the same, so that the cluster zones themselves can be characterised by identical homogenised composite inclusions, called super-inclusion. The calculations at two scales are effectuated, one is macro-composite, in this case the cluster zones are considered to be homogenised composite inclusions (characterised by their effective properties); the other scale is the super-inclusion, which is also a composite. In the super-inclusions the local matrix can have a plastic strain different from the remained matrix. Since the super-inclusions are composite, in order to determine the local stress inside of the super-inclusions, the previous formulation can also be applied. By setting the

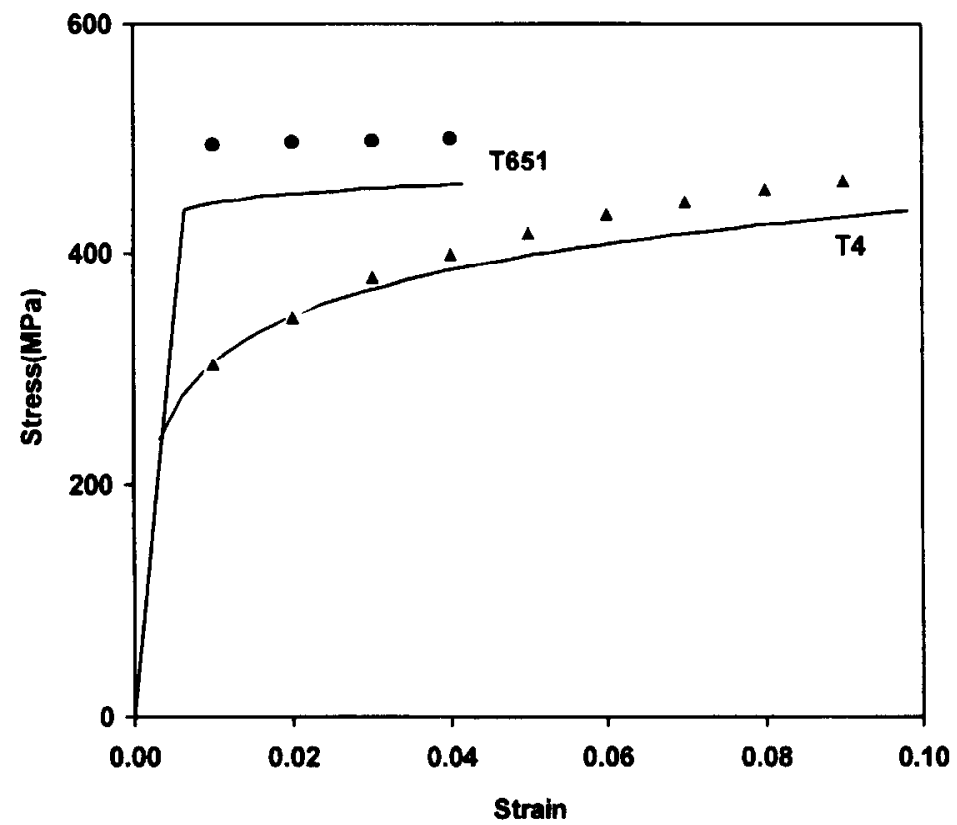

Fig. 2. Comparison between the prediction and the experiment for the stress-strain curves of the composite (solid line: prediction; points: experiment). 


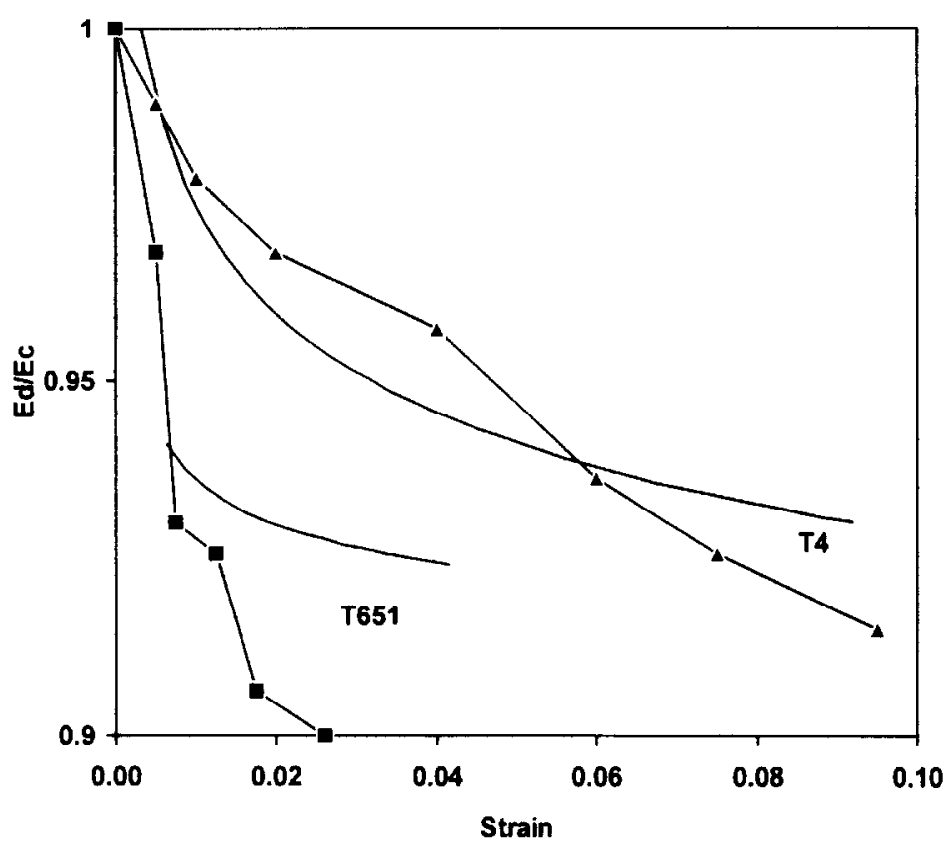

Fig. 3. Comparison between the prediction and the experiment for the modulus variation of the composite (solid line: prediction; points: experiment. $E_{\mathrm{d}}$ : the elastic modulus of damaged composite: $E_{\mathrm{c}}$ : elastic modulus of virgin composite).

homogenised effective stresses for the local matrix inside the super-inclusions and for the rest of the matrix to their current yield stresses, we obtain two equations, which enable us to determine for a given applied stress $\Sigma_{11}$ the corresponding average effective plastic strains in the local matrix inside of super-inclusions and in the rest matrix. The overall properties can be derived with the help of secant moduli method.

In the following computation, the local volume fraction inside the cluster is noted by $c_{3}$, the volume fraction

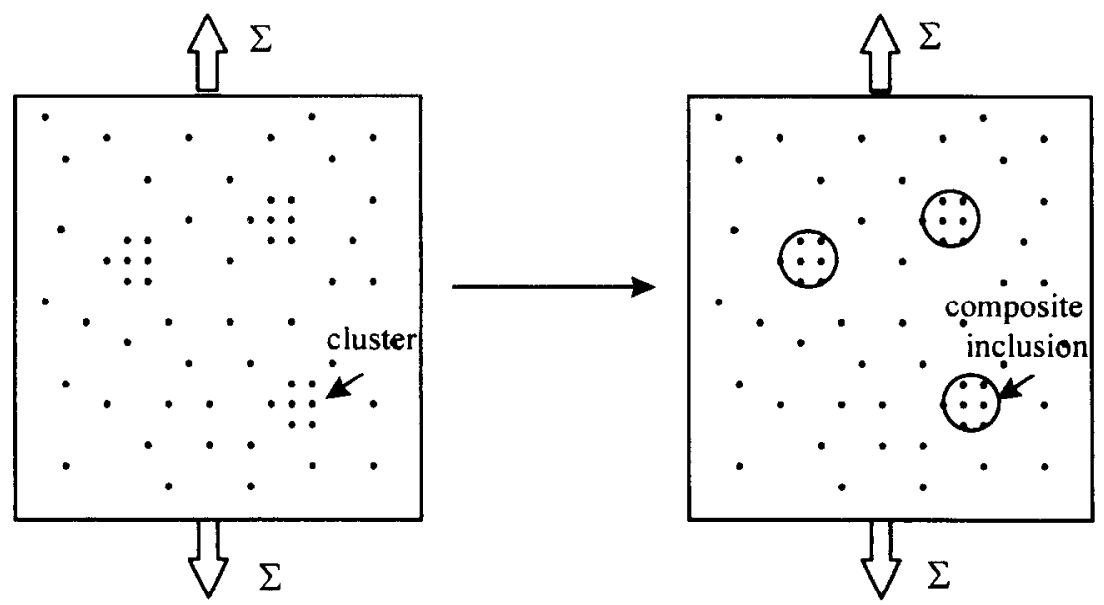

Fig. 4. Particle cluster model 


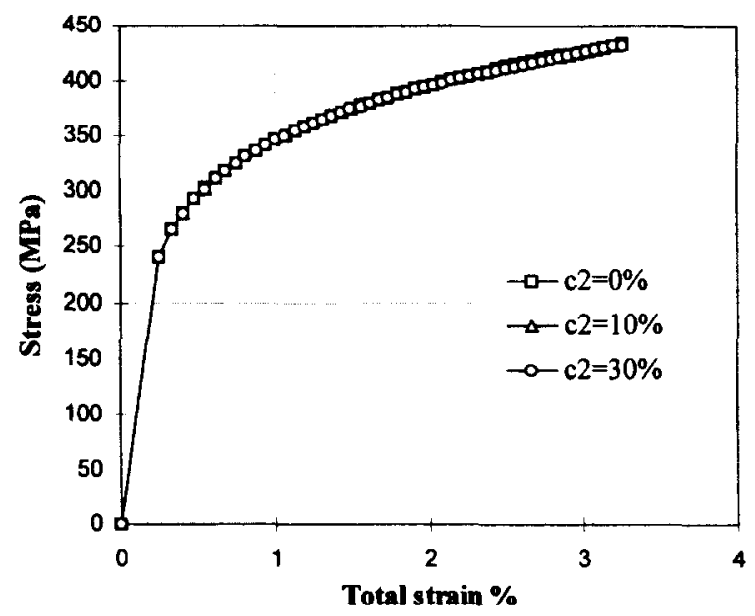

Fig. 5. Influence of the cluster zone on composite stress-strain curves.

of the super-inclusion (cluster) is $c_{2}$ and the remaining particle volume fraction is $c_{1}$. If the total particle volume fraction is noted by $f$, then we have

$$
c_{1}+c_{2}+c_{0}=1, \quad c_{1}+c_{2} c_{3}=f
$$

This concept is applied for the previously studied composites in the T4 condition (without damage) and the results are given in Figs. 5 and 6.

It is seen that the presence of the particle cluster zones has a negligent effect on stress-strain curves for the composite at the same particle volume fraction (Fig. 5). This results in an agreement with those obtained by the finite element calculation performed by Christman et al. [16]. The recent experimental results obtained by Derrien [17] also confirm this prediction. In the case of uniaxial tension, Fig. 6 shows that when the local volume fraction of the particle in the cluster zone is less than that in the remaining region, the stress (in the direction of the tension) bearing on the particle inside the cluster zone is larger than that outside. It is contrary when the local volume fraction of the particle in the cluster zone is larger than that in the rest of the region. This means that in the cluster zone, the particle has less of a probability of being damaged than those outside under

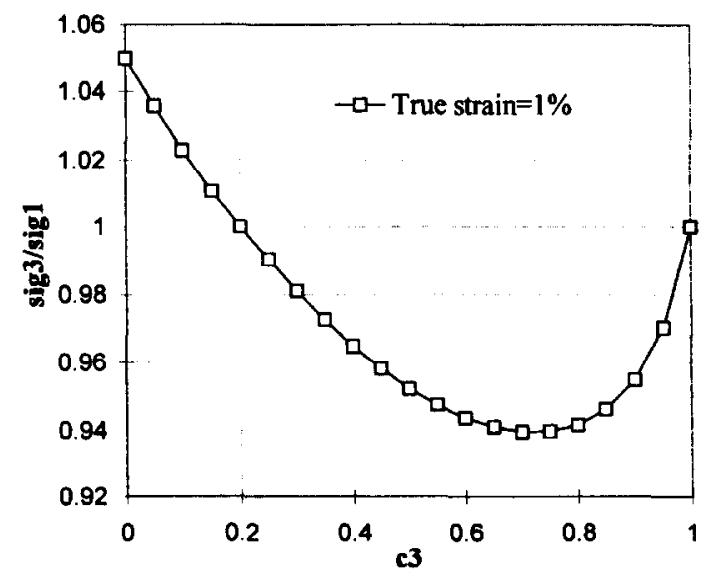

Fig. 6. Comparison of the principle stress in the inclusions inside and outside the cluster zone (sig3: inside; sig 1: outside). 
the same conditions. However in the cluster zone the matrix, which is subjected to an inportant triaxial stress state, may be more sensitive to the void's nucleation and growth. These effects are under investigation.

\section{Discussions and conclusions}

The analytical nature of the proposed method can be easily used to study particular composite systems. For example, if we analyse the influence of the porosity in the matrix on yield potential for a composite reinforced by rigid inclusions (in addition the matrix is assumed to be elastic incompressible), this can be achieved by letting $k_{0} \rightarrow \infty, \mu_{2}=k_{2}=0$ and $\mu_{1} \rightarrow \infty, k_{1} \rightarrow \infty$ and setting the defined homogenised matrix effective stress (Eq. (3)) to the yielding stress of the matrix, this leads to the following yielding potential for this composite system

$$
\frac{12 c_{0}^{2}+15 c_{1} c_{2}+10 c_{0}\left(3 c_{1}+2 c_{2}\right)}{3 c_{0}\left(2 c_{0}+5 c_{1}\right)^{2}} \Sigma_{e}^{2}+\frac{9}{4} \frac{c_{2}}{c_{0}\left(1-c_{2}\right)} \Sigma^{2}-\sigma_{y}^{2}=0
$$

In this case $c_{0}$ is volume fraction of the matrix, $c_{1}$ is the volume fraction of the rigid particle and $c_{2}$ is the volume fraction of the porosity in the matrix. If the volume fraction of the rigid inclusion vanishes, Eq. (13) naturally yields the results for porous material given by Hu [13] and Qiu and Weng [11].

To conclude, we therefore propose a general analytical model for a composite with isotropic phases and two populations of spherical inclusions. The plastic deformation of the matrix is taken into account with secant moduli method and a homogenised matrix effective stress defined from the average of the local effective stress over the matrix. The general analytical formulation was then applied to analyse the influence of particle fracture and particle cluster on the composite overall properties. The model can account quantitatively for the influence of particle fracture on the mechanical properties of PMMCs. Application of the proposed model to the particle cluster case shows that the particle cluster has little influence on the elastoplastic strain and stress curves of the composite.

\section{Appendix A. Mori-Tanaka's mean field theory}

Consider a representative volume element (RVE), with two populations of particles homogeneously dispersed in the matrix. All phases are isotropic and elastic, so the composite as a whole is also isotropic. $L_{0}, L_{1}$ and $L_{2}$ denote the stiffness tensors for the matrix, particle of population one and two, respectively, their volume fractions are $c_{0}, c_{1}$ and $c_{2}$. A macroscopic stress $\Sigma$ is applied along the boundary of RVE. Taking the matrix as the comparison material, $\boldsymbol{E}_{0}$ is defined by $\Sigma=\boldsymbol{L}_{0} \boldsymbol{E}_{0}$ and the matrix average stress in the composite is given by $\sigma_{0}=\boldsymbol{L}_{0}\left(\boldsymbol{E}_{0}+\varepsilon_{\mathrm{p} 0}\right)$ and the average strain in the matrix is $\varepsilon_{0}=\boldsymbol{E}_{0}+\varepsilon_{p 0}$. The average strains in the inclusions of population one and two differ further from the matrix average strain by $\varepsilon_{\mathrm{p} 1}$ and $\varepsilon_{\mathrm{p} 2}$, so that the average stresses of the inclusions of population one and two are

$$
\begin{aligned}
& \sigma_{1}=L_{1}\left(E_{0}+\varepsilon_{\mathrm{p} 0}+\varepsilon_{\mathrm{p} 1}\right)=\boldsymbol{L}_{0}\left(\boldsymbol{E}_{0}+\varepsilon_{\mathrm{p} 0}+\varepsilon_{\mathrm{p} 1}-\varepsilon_{1}^{*}\right) \\
& \sigma_{2}=L_{2}\left(E_{0}+\varepsilon_{\mathrm{p} 0}+\varepsilon_{\mathrm{p} 2}\right)=L_{0}\left(E_{0}+\varepsilon_{\mathrm{p} 0}+\varepsilon_{\mathrm{p} 2}-\varepsilon_{2}^{*}\right)
\end{aligned}
$$

and we have

$$
\varepsilon_{\mathrm{p} 1}-\boldsymbol{S}_{\varepsilon_{1}}^{*}, \quad \varepsilon_{\mathrm{p} 2}=\boldsymbol{S}_{\varepsilon_{2}^{*}}^{*}
$$

$S$ being the Eshelby tensor. Using the fact that $c_{0} \sigma_{0}+c_{1} \sigma_{1}+c_{2} \sigma_{2}=\Sigma$, we get

$$
\varepsilon_{\mathrm{p} 0}+c_{1}\left(\varepsilon_{\mathrm{p} 1}-\varepsilon_{1}^{*}\right)+c_{2}\left(\varepsilon_{\mathrm{p} 2}-\varepsilon_{2}^{*}\right)=0
$$


From Eqs. (A.1), (A.2) and (A.3), the unknown quantities $\varepsilon_{\mathrm{p} 1}, \varepsilon_{\mathrm{p} 2} \varepsilon_{\mathrm{p} 0}, \varepsilon_{1}^{*}$ and $\varepsilon_{2}^{*}$ can be evaluated, the composite compliance tensor can then be calculated. For spherical inclusions and isotropic phases, Eqs. (A.1), (A.2) and (A.3) can be written in deviatoric and spherical parts, solving the previous equations leads to the results given in Section 2 .

\section{Appendix B}

The expression of the constants are:

$$
\begin{aligned}
h_{0}= & c_{0} \mu_{0}^{4}\left(9 k_{0}+8 \mu_{0}\right)^{2}\left[3 k_{0}\left(5-2 c_{0}\right)\left(9 k_{0}+18 \mu_{0}\right)+32\left(5-3 c_{0}\right) \mu_{0}^{2}\right] \\
h_{1}= & 12 c_{0} \mu_{0}^{3}\left(9 k_{0}+8 \mu_{0}\right)\left[27\left(3 c_{0}+5 c_{2}\right) k_{0}^{3}+6\left(51-36 c_{1}+34 c_{2}\right) k_{0}^{2} \mu_{0}+16\left(22-17 c_{1}+18 c_{2}\right) \mu_{0}^{2} k_{0}\right. \\
& \left.+64\left(2 c_{0}+5 c_{2}\right) \mu_{0}^{3}\right] \\
h_{3}= & 12 \mu_{0}^{2}\left[\left(12\left(c_{1}-1\right)^{2}+12\left(c_{2}-1\right)^{2}-c_{2} c_{1}-12\right)\left(81 k_{0}^{4}+256 \mu_{0}^{4}\right)+\left(78-141\left(c_{1}+c_{2}\right)+c_{1} c_{2}\right.\right. \\
& \left.\left.+63\left(c_{1}^{2}+c_{2}^{2}\right)\right)\left(72 k_{0}^{3} \mu_{0}+128 k_{0} \mu_{0}^{3}\right)+48\left(241-417\left(c_{1}+c_{2}\right)+2 c_{1} c_{2}+176\left(c_{1}^{2}+c_{2}^{2}\right)\right) k_{0}^{2} \mu_{0}^{2}\right] \\
h_{4}= & 6 \mu_{0}^{2}\left[81\left(3 c_{0}+5 c_{2}\right)\left(2 c_{0}+5 c_{1}\right) k_{0}^{4}+72\left(3 c_{0}+5 c_{2}\right)\left(13 c_{0}+25 c_{1}\right) k_{0}^{3} \mu_{0}-24\left(174\left(c_{1}-c_{2}\right)^{2}\right.\right. \\
& \left.-4 c_{1} c_{2}+67\left(c_{1}+c_{2}\right)-241\right) k_{0}^{2} \mu_{0}^{2}+128\left(13 c_{0}+25 c_{2}\right)\left(3 c_{0}+5 c_{1}\right) k_{0} \mu_{0}^{3} \\
& +256\left(2 c_{0}+5 c_{2}\right)\left(3 c_{0}+5 c_{1}\right) \mu_{0}^{4} \\
h_{5}= & 12 \mu_{0}^{2}\left[\left(12\left(c_{1}-1\right)^{2}+12\left(c_{2}-1\right)^{2}-c_{2} c_{1}-12\right)\left(81 k_{0}^{4}+256 \mu_{0}^{4}\right)+\left(78-141\left(c_{1}+c_{2}\right)+c_{1} c_{2}\right.\right. \\
& \left.\left.+63\left(c_{1}^{2}+c_{2}^{2}\right)\right)\left(72 k_{0}^{3} \mu_{0}+128 k_{0} \mu_{0}^{3}\right)+48\left(241-417\left(c_{1}+c_{2}\right)+2 c_{1} c_{2}+176\left(c_{1}^{2}+c_{2}^{2}\right)\right) k_{0}^{2} \mu_{0}^{2}\right] \\
h_{6}= & 72 c_{0} \mu_{0}\left(k_{0}+2 \mu_{0}\right)\left[27\left(2 c_{0}+5 c_{1}\right) k_{0}^{3}+12\left(22+18 c_{1}-17 c_{2}\right) k_{0}^{2} \mu_{0}+8\left(51+34 c_{1}-36 c_{2}\right) \mu_{0}^{2} k_{0}\right. \\
& \left.+64\left(3 c_{0}+5 c_{1}\right) \mu_{0}^{3}\right] \\
h_{8}= & 261 c_{0}\left(k_{0}+2 \mu_{0}\right)^{2}\left[3\left(5-3 c_{0}\right) k_{0}^{2}+8\left(5-2 c_{0}\right) k_{0} \mu_{0}+8\left(5-2 c_{0}\right) \mu_{0}^{2}\right]
\end{aligned}
$$

$h_{2}$ has the same expression as $h_{1}$ with the inter-change of $c_{1}$ and $c_{2}$, the same for $h_{4}$ and $h_{5}, h_{7}$ and $h_{6}$ and

$$
\begin{aligned}
& g_{0}=192 c_{0}\left(1-c_{0}\right) \mu_{0}^{4}, \\
& g_{1}=96 c_{0} \mu_{0}^{3}\left(3 c_{2}-4 c_{1} \mu_{0} / k_{0}\right), \\
& g_{3}=-24 \mu_{0}^{2}\left[24\left(c_{0}\left(1-c_{0}\right)+c_{1} c_{2}\right) \mu_{0} / k_{0}+c_{1} c_{2}\left(16\left(\mu_{0} / k_{0}\right)^{2}+9\right)\right] \\
& g_{4}=12 \mu_{0}^{2}\left[9 c_{2}\left(1-c_{2}\right)+24 c_{1} c_{2} \mu_{0} / k_{0}+16 c_{1}\left(1-c_{1}\right)\left(\mu_{0} / k_{0}\right)^{2}\right] \\
& g_{6}=72 c_{0}\left(4 c_{1} \mu_{0}-3 c_{2} k_{0}\right)\left(\mu_{0} / k_{0}\right)^{2} \\
& g_{8}=108 c_{0}\left(1-c_{0}\right)\left(\mu_{0} / k_{0}\right)^{2}
\end{aligned}
$$

$g_{2}$ has the same expression as $g_{1}$ with the inter-change of $c_{1}$ and $c_{2}$, the same for $g_{4}$ and $g_{5}, g_{7}$ and $g_{6}$.

\section{References}

[1] D.J. Lloyd, Int. Mater. Rev. 39 (1994) 1-23.

[2] G. Bao, J.W. Hutchinson, R.M. McMecking, Acta Metall. Mater. 39 (1991) 1871-1882. 
[3] M. Taya, K.E. Lulay, D.J. Lloyd, Acta Metall. Mater. 39 (1991) 73-87.

[4] J. Llorca, A. Martin, J. Ruiz, M. Elices, Metal. Trans. A 24 (1993) 1575-1588.

[5] M. Berveiller, A. Zaoui, J. Mech. Phys. Solids 26 (1979) 325-344.

[6] G.P. Tandon, G.J. Weng, ASME J. Appl. Mech. 55 (1988) 126 135.

[7] N. Bourgeous, Ph.D. Thesis, Ecole Centrale Paris, 1994.

[8] G.K. Hu, The Second Asia-Pacific Symposium on Advances in Engineering Plasticity and its Applications, Int. Academic Pub., 1994, pp. $107-112$.

[9] J. Llorca, P. Poza, Acta Metall. Mater. 43 (1995) 3959-3969.

[10] D.J. L.lord, Acta Metall. Mater. 39 (1991) $59 \ldots 71$.

[11] Y.P. Qiu, G.J. Weng, ASME J. Appl. Mech. 59 (1992) $261-268$.

[12] G.K. Hu, Int. J. Plasticity 12 (1996) 439-449.

[13] G.K. Hu, Int. J. Solids Struct. 34 (1997) $1007-1015$.

[14] T. Mori, K. Tanaka, Acta Metall. Mater. 21 (1973) $571-574$.

[15] G. Guo, G.K. Hu, J.B. Bai, to be submitted.

[16] T. Christman, A. Needleman, S. Suresh, Acta Metall. Mater. 37 (1989) 3029-3050.

[17] K. Derrien, Ph.D. Thesis, Ecole Nationale Superieure d'Arts et Metiers, 1997. 